\title{
Mechanical force involved multiple fields switching of both local ferroelectric and magnetic domain in a $\mathrm{Bi}_{5} \mathrm{Ti}_{3} \mathrm{FeO}_{15}$ thin film
}

\author{
Tingting Jia ${ }^{1}$, Hideo Kimura ${ }^{1}$, Zhenxiang Cheng ${ }^{2}$, Hongyang Zhao ${ }^{3}$, Yoon-Hyun Kim ${ }^{1}$, Minoru Osada ${ }^{1}$, \\ Takao Matsumoto ${ }^{4}$, Naoya Shibata ${ }^{4}$ and Yuichi Ikuhara ${ }^{4}$
}

Multiferroics have received intense attention due to their great application potential in multi-state information storage devices and new types of sensors. Coupling among ferroic orders such as ferroelectricity, (anti-)ferromagnetism, ferroelasticity and so on will enable dynamic interaction between these ordering parameters. Direct visualization of such coupling behavior in singlephase multiferroic materials is highly desirable for both applications and fundamental study. Manipulation of both ferroelectric and magnetic domains of $\mathrm{Bi}_{5} \mathrm{Ti}_{3} \mathrm{FeO}_{15}$ thin film using electric field and external mechanical force is reported, which confirms the magnetoelectric coupling in $\mathrm{Bi}_{5} \mathrm{Ti}_{3} \mathrm{FeO}_{15}$, indicates the electric and magnetic orders are coupled through ferroelasticity. Due to the anisotropic relaxation of ferroelastic strain, the back-switching of out-of-plane electric domains is not as obvious as in-plane. An inevitable destabilization of the coupling between elastic and magnetic ordering happens because of the elastic strain relaxation, which result in a subsequent decay of magnetic domain switching. Mechanical force applied on the surface of $\mathrm{Bi}_{5} \mathrm{Ti}_{3} \mathrm{FeO}_{15}$ film generates by an atomic force microscopy tip will effectively drive a transition of the local ferroelastic strain state, reverse both the polarization and magnetization in a way similar to an electric field. Current work provides a framework for exploring cross-coupling among multiple orders and potential for developing novel nanoscale functional devices.

NPG Asia Materials (2017) 9, e349; doi:10.1038/am.2017.3; published online 17 February 2017

\section{INTRODUCTION}

Multiferroic materials have been drawing extensive attention worldwide because they simultaneously possess ferroelectric, magnetic and/or ferroelastic properties. The coupling between these properties enables the dynamic manipulation of one ordering parameter by another, which is promising for broad applications in sensing, actuation, memory and so on. ${ }^{1-3}$ Numerous efforts have been made to investigate the coupling phenomena, especially the coupling between electric and magnetic orderings that would account for the magnetoelectric (ME) effect in multiferroic materials, due to the rich fundamental physics and exciting application potential in multiply controlled devices. ${ }^{3,4}$ Recently, the development of scanning probe microscopy (SPM) techniques has enabled us to not only directly visualize but also switch both ferroelectric domains and magnetic domains. ${ }^{5,6}$ Therefore, the direct visualization of the coupling between magnetic domains and ferroelectric domains has been made possible and recently has become a powerful tool for ME studies. Zhao et al. ${ }^{7}$ observed antiferromagnetic domain switching induced by ferroelectric polarization switching in $\mathrm{BiFeO}_{3}$ thin films, whereas Keeney et al. ${ }^{8}$ demonstrated that ferroelectric domain polarization switching could be induced by an applied magnetic field in Aurivillius-phase multiferroic thin films. These works have demonstrated the powerful capability of SPM technology in the study of ME coupling. Furthermore, investigations on the nanoscale ferroelectric domain switching under mechanical force loaded by a SPM tip is drawing increasing attention. ${ }^{9,10}$ Recently, mechanical-force-induced ferroelectric domain switching in multiferroics has been reported by several groups. ${ }^{11-13}$ It is natural to ask whether such an external mechanical force could switch two or all three types of ferroic domains (ferroelectric, ferroelastic and magnetic domains) in multiferroic materials. Finding the answer in multiferroics is of fundamental and practical importance for understanding their quasistatic and dynamic behavior in multiply controlled devices applications.

Zhao et al. ${ }^{14}$ found that strong ME coupling in pure $\mathrm{Bi}_{5} \mathrm{FeTi}_{3} \mathrm{O}_{15}$ (BTF) film at room temperature (RT), which gives us a new way of achieving ME coupling in ferroelectric materials without long-range magnetic ordering. However, the mechanism of ME switching in BTF has not been clarified, or rather the switching path of the electric and magnetic orders is still unknown. BTF has a structure with fourlayered pseudoperovskite units of $\left(\mathrm{Bi}_{3} \mathrm{Ti}_{3} \mathrm{FeO}_{13}\right)^{2-}$, sandwiched by two

\footnotetext{
${ }^{1}$ Research Center for Functional Materials, National Institute for Materials Science, Ibaraki, Japan; ${ }^{2}$ Institute for Superconducting \& Electronic Materials, University of Wollongong, Innovation Campus, North Wollongong, NSW, Australia; ${ }^{3}$ Department of Materials Science and Engineering, Wuhan Institute of Technology, Wuhan, China and ${ }^{4}$ Institute of Engineering Innovation, School of Engineering, The University of Tokyo, Tokyo, Japan

Correspondence: Dr T Jia or Dr H Kimura, Environment and Energy Materials Research Division, National Institute for Materials Science, 1-2-1 Sengen, Tsukuba, Ibaraki 305-0047, Japan.

E-mail: jia.tinting@nims.go.jp or kimura.hideo@nims.go.jp

or Professor Z Cheng, Institute for Superconducting \& Electronic Materials, University of Wollongong, Innovation Campus, North Wollongong, New South Wales 2500, Australia. E-mail: cheng@uow.edu.au

Received 7 April 2016; revised 24 October 2016; accepted 20 November 2016
} 

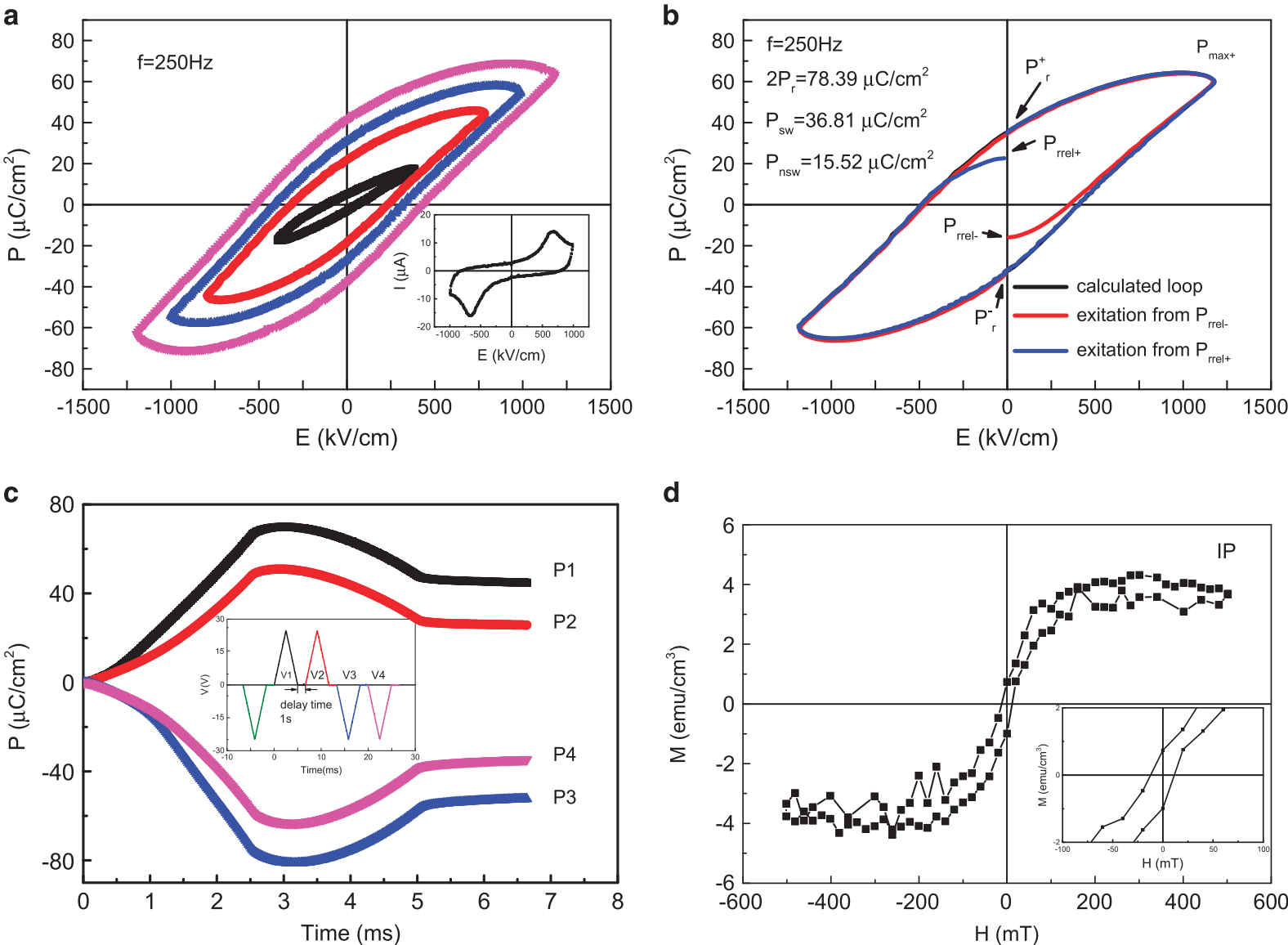

Figure 1 Ferroelectric dynamic hysteresis measurement and magnetic hysteresis loop. (a) Electric field dependence of ferroelectric polarization ( $P-E$ loops) of the $\mathrm{Bi}_{5} \mathrm{FeTi}_{3} \mathrm{O}_{15}$ (BTF) thin film measured at $250 \mathrm{~Hz}$, inset shows the correspond $I-V$ curve; (b) a giant remanent polarization of $2 P_{\mathrm{r}}=\left|P_{\mathrm{r}}^{+}\right|+\left|P_{\mathrm{r}}^{-}\right|=78.39 \mu \mathrm{C} \mathrm{cm}-2$ is obtained by bipolar excitation hysteresis measurement at $250 \mathrm{~Hz}$. Triangular voltage excitation was applied on the sample. The red loop start from negative relaxed remanent polarization state $\left(P_{\text {rrel- }}\right)$ then goes to the positive saturation $\left(P_{\text {max }}\right)$ and end at the positive remanent polarization state $\left(P_{\mathrm{r}}^{+}\right)$. The blue loop start from the positive relaxed remanent polarization state $\left(P_{\text {rrel }+}\right)$ and turns into the negative remanent polarization state $\left(P_{\mathrm{r}}^{-}\right)$. The switching polarization $P_{\mathrm{sw}}=P_{\mathrm{max}+}-P_{\text {rrel }-}$, and the non-switching polarization $P_{\mathrm{nsw}}=P_{\text {max }}-P_{\text {rrel+. }}$ (c) Positive-up-negative-down (PUND) switching polarization as a function of voltage, which proves intrinsic ferroelectricity in the film, inset is the waveform of the applied triangle pulse: the write pulse rise-time is $2.5 \mathrm{~ms}$, and the read pulse delay is $1 \mathrm{~s}$; (d) in-plane magnetic hysteresis $(M-H)$ loop measured at room temperature, with the inset showing the enlarged central part of the $M-H$ loop of the BTF thin film.

$\left(\mathrm{Bi}_{2} \mathrm{O}_{2}\right)^{2+}$ slabs along the $c$ axis ${ }^{15}$ (shown in Supplementary Figure $\mathrm{S} 1$ ). Owing to its unique crystal structure, BTF has a high ferroelectric transition Curie point $\left(T_{\mathrm{C}}\right)$ of $750{ }^{\circ} \mathrm{C}$ (with a structural transition from the $A 2_{1}$ am structure to the $I 4 / \mathrm{mmm}$ structure) and large spontaneous polarization. ${ }^{16,17}$ The strong ferroelectricity is attributed to the rotation and tilt of the $\mathrm{Ti}(\mathrm{Fe})-\mathrm{O}$ octahedra in the perovskite layers, where lone pair electrons of $\mathrm{Bi}^{3+}$ ions induce $\mathrm{Ti}(\mathrm{Fe})$ ions to deviate from the center of the $\mathrm{Ti}(\mathrm{Fe})-\mathrm{O}$ octahedra along the $a$ axis, ${ }^{18}$ while the $\left(\mathrm{Bi}_{2} \mathrm{O}_{2}\right)^{2+}$ slabs play an important role in space-charge compensation and insulation. ${ }^{19,20}$ Although BTF is attracting more attention as a promising candidate for single-phase RT multiferroics and thus for ME coupling, ${ }^{14,21,22}$ it is reported to have an antiferromagnetic ordering with a Néel transition temperature $\left(T_{\mathrm{N}}\right)$ of $80 \mathrm{~K} .{ }^{23}$ Therefore, it is obvious from the above reports that the origin of the ME coupling at RT in BTF has yet to reach an agreement, let alone manipulation of nanoscale domains using electric field, magnetic field and external mechanical force.

In this work, $\mathrm{Bi}_{5} \mathrm{FeTi}_{3} \mathrm{O}_{15}$ thin films were deposited on $\mathrm{Pt} / \mathrm{TiO}_{2} / \mathrm{SiO}_{2} / \mathrm{Si}$ substrates using pulsed laser deposition. An orthorhombic structure with space group $A 2{ }_{1} a m$ (36) was indexed by X-ray diffraction as shown in Supplementary Figure S1. The detailed microstructure and component analysis were carried out by transmission and scanning electron microscopy (Supplementary Figure S2). We have visualized the switching of both ferroelectric and magnetic domains by electrical and magnetic field using a SPM system. The time decay feature of magnetic domain switching accompanying ferroelectric domain switching is revealed after removing the electrical field, which is in good agreement with the nature of short-range magnetic ordering in BTF film. More importantly, we were successful in switching ferroelectric domains, as well as magnetic domains, by using a mechanical force applied on the probe tip of the SPM system. We believe that similar phenomena can be observed in other typical multiferroic materials. Therefore, our findings open up a new route to manipulate the magnetism in multiferroics by mechanical force and broaden the range of applications for future design of multifunctional devices made from multiferroic materials.

\section{EXPERIMENTAL PROCEDURES}

\section{Film deposition}

$\mathrm{Bi}_{5} \mathrm{Ti}_{3} \mathrm{FeO}_{15}$ (BTF) thin film was deposited on $\mathrm{Pt} / \mathrm{TiO}_{2} / \mathrm{SiO}_{2} / \mathrm{Si}$ substrates by a pulsed laser deposition system with the laser source at $355 \mathrm{~nm}$ and a repetition rate of $10 \mathrm{~Hz}$. The ceramic target for BTF deposition was prepared using a 

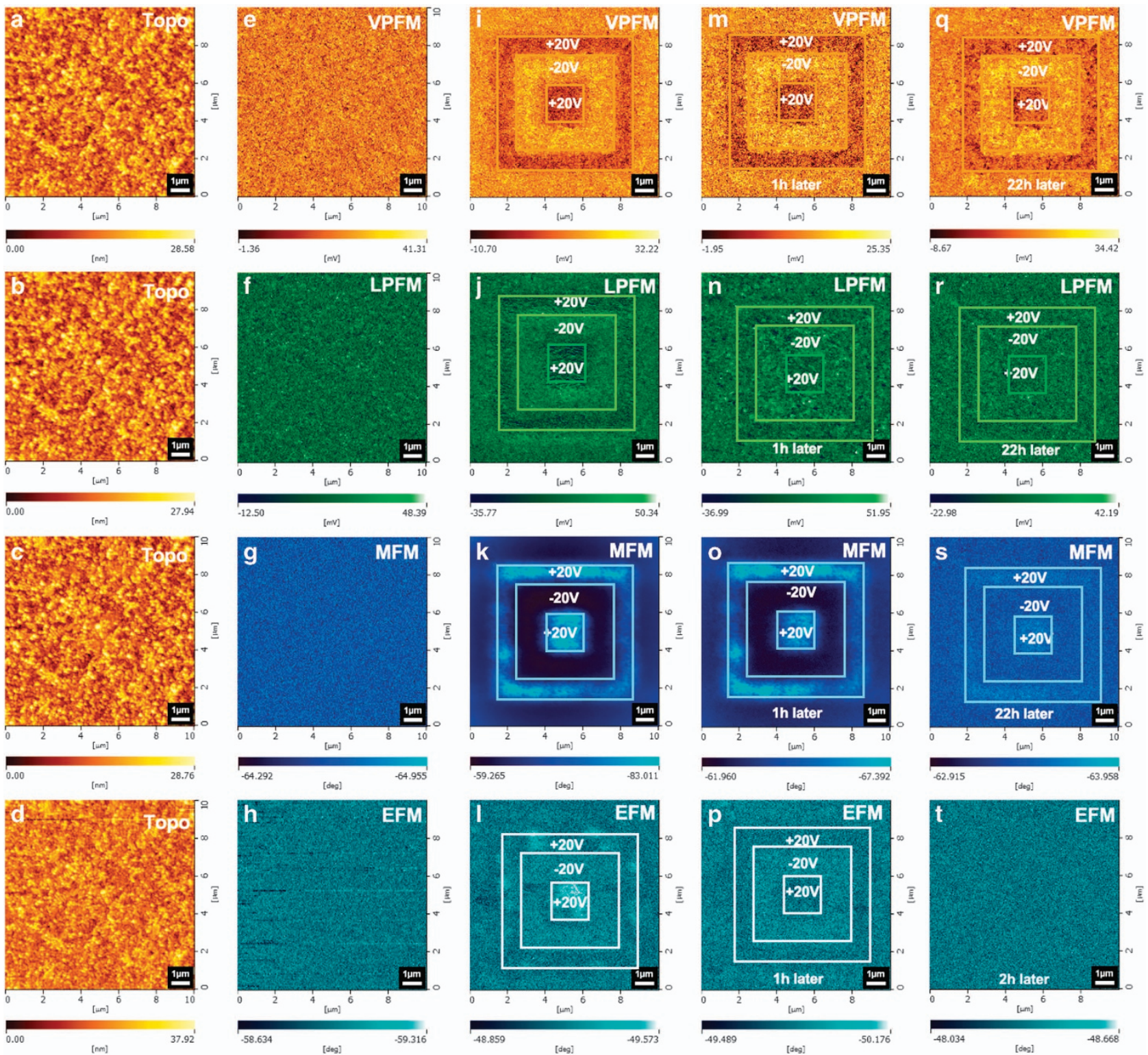

Figure 2 Reversible control of both ferroelectric and magnetic domains by electric field (E). (a-d) Topographic images; (e-h) vertical piezoresponse force microscope (VPFM) image, lateral piezoresponse force microscope (LPFM) image, magnetic force microscope (MFM) phase image and corresponding electrostatic force microscope (EFM) image of the $\mathrm{Bi}_{5} \mathrm{FeTi}_{3} \mathrm{O}_{15}$ (BTF) thin film before electric poling. (i) VPFM image poled by $+20 \mathrm{~V}$ in the area of $7 \times 7 \mu \mathrm{m}^{2},-20 \mathrm{~V}$ in $5 \times 5 \mu \mathrm{m}^{2}$ and $+20 \mathrm{~V}$ in $2 \times 2 \mu \mathrm{m}^{2}$, with reading over $10 \times 10 \mu \mathrm{m}^{2}$. (j) Corresponding LPFM image; (k) MFM phase image and (I) EFM. $(\mathbf{m}-\mathbf{t})$ Evolution of VPFM, LPFM, MFM and EFM images of the poled BTF thin film collected after removing the electric field.

conventional solid-state reaction process. BTF thin films were deposited at $470-500{ }^{\circ} \mathrm{C}$ for $1 \mathrm{~h}$, followed by an in situ thermal annealing process for $30 \mathrm{~min}$ and then cooled down to RT. The film thickness is $\sim 269 \mathrm{~nm}$ (Supplementary Figure S2).

\section{Macroscale electrical and magnetic measurements}

Pt top electrodes were coated on the surface of the BTF thin film through a shadow mask with a diameter of $100 \mu \mathrm{m}$ to form a capacitor. Dynamic hysteresis measurements were carried out to verify the ferroelectricity using an aixACCT TF-1000 ferroelectric tester (aixACCT Systems GmbH, Aachen, Germany). (Figure 1a-c). Magnetic properties of the as-deposited BTF film were measured using a superconducting quantum interference device (Quantum Design MPMS magnetometer, Quantum Design Japan, Tokyo, Japan). Here the diamagnetic moment of the substrate was calculated from measurements on the bare substrate and subtracted from the raw data (Figure 1d).

\section{SPM measurement}

A Nanocute SPI 3800 SPM system and an E-sweep SPM system (Hitachi HiTech Science, Tokyo, Japan), which enable piezoresponse force microscopy (PFM), electrostatic force microscope (EFM) and magnetic force microscopy (MFM) measurements, were used to investigate local piezoelectric/ferroelectric and magnetic properties at RT. A conduction tip (Si cantilever coated with Rh) was used to apply electric field $(E)$ and mechanical force $(F)$ in contact mode PFM measurements of which the spring constant was $15 \mathrm{~N} \mathrm{~m}^{-1}$, and the resonance frequency was $139 \mathrm{kHz}$. In PFM measurements, Acos images were recorded together with topographic images, applying $5 \mathrm{kHz}$ and $2 \mathrm{~V}$ oscillation under $\mathrm{a} \pm 20 \mathrm{~V}$ d.c. bias. The $A \cos$ image correspondes to $A \cos (\theta+\omega t)$, where $A$ is the amplitude, $\theta$ is the phase shift between the driving voltage $V_{\text {a.c. }}$ and the voltage induced deformation, $\omega$, represents the frequency and $t$ is the sample thickness. After the electrical and mechanical poling, the vertical piezoresponse force microscope (VPFM) and lateral piezoresponse force microscope (LPFM) 
images were read with an a.c. voltage of $4 \mathrm{~V}$ and $5 \mathrm{kHz}$ without applying any d.c. bias. In order to reduce the effects of injected charges as much as possible during probing, we blew on the sample for $2 \mathrm{~min}$ using a static eliminator fan (HAKKO FE-510, Osaka, Japan) between each writing process. The displacement-voltage curve was obtained directly by applying d.c. voltage on the film surface in PFM mode. (Supplementary Figure S3). Mechanical force was applied on the film surface by loading mechanical force on the tip during scanning. A magnetic tip coated with CoPtCr film was used in the MFM measurements. The reason for not using the MFM tip to do force scanning was to avoid the magnetization effect from the very weak magnetic field of the MFM tip. In MFM measurements, the trace is first recorded in tapping mode to image the topography of the surface; and the second step is in a lift mode in which the tip does not touch the film surface to assess the stray magnetic field perpendicular to the surface. To avoid the contribution of the interaction to the tip-sample force in $M F M,{ }^{24}$ the tip-sample distance was kept at $10 \mathrm{~nm}$ to achieve a balance between avoiding the van der Waals contribution and improving the magnetic response. At this distance, the sample generates stronger stray fields, and the magnetic interaction force dominates, so the electrostatic force can be neglected. ${ }^{25}$ Polarization reversal was also confirmed by scanning nonlinear dielectric microscopy, as shown in Supplementary Figure S5.

\section{RESULTS AND DISCUSSION}

\section{Domain switching by electric field}

Figure $2 \mathrm{a}-\mathrm{h}$ is topographic images and corresponding VPFM, LPFM, MFM and EFM images before electrical switching. Figure $2 \mathrm{i}$ shows a box-in-box VPFM pattern of BTF thin film after electrically poling. The dark and bright regions correspond to ferroelectric domain state with $+\mathrm{P}$ and $-\mathrm{P}$, respectively, indicating that the as-deposited thin film can be reversibly switched between two ferroelectric state. The VPFM shows the information from out-of-plane (OP) polarization component, while the LPFM provides information on the in-plane (IP) polarization component (schematic illustration as shown in Supplementary Figure S7). Figure $2 \mathrm{j}$ shows a similar lithographic pattern, indicating that the perpendicular electric field could also partially switch the IP polarization. The VPFM and LPFM images present two components of the polarization vector, ${ }^{26}$ corresponding to the orthogonal components to the $a$ axis of the as-deposited BTF thin film along the OP and IP directions. As the ME coefficient in the BTF thin film is $400 \mathrm{mV}$ per $(\mathrm{Oe} \cdot \mathrm{cm})$ at $\mathrm{RT},{ }^{14}$ it is possible to switch magnetic domains in this material by applying electric field $(\boldsymbol{E})$. Figure $2 \mathrm{k}$ shows the corresponding MFM phase image in the same area after electric poling. A sharp contrast in the magnetic domains corresponding to the ferroelectric domains was observed, indicating that magnetic domains in BTF thin film were switched by E. From the above measurements, we directly visualized the local ME coupling in BTF thin film at RT. There are two mechanisms for ME coupling that could be considered: an $E$ shifts the positions of the Fe-ions relative to the O-ions, changing the dipolar and exchange contributions to the magnetic interactions and modifying the electronic wavefunctions at the same time, which thus changes the magnetic coupling. ${ }^{27}$ Another mechanism is that the $E$ causes ferroelastic strain in the film, which could be mechanically transferred to the magnetic component, where it modifies the local magnetization of the domain structures. ${ }^{28}$ In the pseudoperovskite layer of BTF thin film, there are two types of atomic position for $\mathrm{Ti}$ and/or $\mathrm{Fe}$ with respect to four oxygen octahedron identified by direct adjacent to $\mathrm{Bi}_{2} \mathrm{O}_{2}$ layer or far from $\mathrm{Bi}_{2} \mathrm{O}_{2}$ layer in BTF with space group $\mathrm{A} 2{ }_{1} \mathrm{am} .{ }^{29}$ The occupancy of both sites by Fe or $\mathrm{Ti}$ atoms in the compounds is random and almost equal in general. ${ }^{30}$ According to the Mössbauer spectra, there is no long-range magnetic ordering in BTF. ${ }^{14}$ However, weak magnetic hysteresis is observed at RT. For our sample, the shape of magnetic-temperature curve indicated a generic feature of magnetic disordered system due to random distribution of the Fe spins on available sites. ${ }^{14}$ It is possibly due to $\mathrm{Fe}$ atoms randomly substitute $\mathrm{Ti}$ atoms in the pseudoperovskite layers of BTF. Generally, there is a charge disproportionation of Fe at octahedral coordinated site and the tetrahedral site. ${ }^{30}$ Although the macroscopy analysis indicated the distribution of $\mathrm{Fe}$ and $\mathrm{Ti}$ atoms are random and almost equal in $\mathrm{BTF}^{31}$ a small inhomogeneous $\mathrm{Fe}$ distribution in a nano-region still persist, which will lead to a shortrange magnetic ordering in BTF thin film. An $E$ causes ferroelastic strain in the film and shifts the positions of the Fe-ions relative to the $\mathrm{Ti}$ and/or O-ions, changing the dipolar and exchange contributions to the magnetic interactions and modifying the electronic wavefunctions at the same time, which thus changes the magnetic coupling. ${ }^{27}$ For multiaxial ferroelectrics as BTF materials, the ferroelectric domains are always coupled to ferroelastic domains in particular orientation. Once the ferroelectric domain is switched by $E$, the local ferroelastic strain state in the film is simultaneously rearranged. The ferroelastic domain is switched to a high-energy state accompanied with the polarization switching. Once $E$ was removed, a relaxation of the switched ferroelastic domain back to the original ferroelastic state will happen. The ferroelastic domains could either switch to the state antiparallizing to the ferroelectric polarization or transited along a different distortion axis. Further investigation of the evolution of ferroelectric and magnetic domains after electric field switching was carried out. After removing the $E$ for $1 \mathrm{~h}$, no obvious change was observed in either of the VPFM and MFM images (Figure $2 \mathrm{~m}$ and $\mathrm{o}$ ), but the switched pattern in the LPFM image became very weak (Figure 2n). It is obvious that the relaxation of ferroelastic domain is anistropic, along the OP and IP directions, due to the inhomogeneous feroelastic strain distribution in the BTF thin film. The IP ferroelastic relaxation is more effective and faster than OP. In an aurivillius layered structure such as BTF, there are four spontaneous polarizations along the $\langle 100\rangle$ directions in the pseudoproveskite layer. Only the $180^{\circ}$ ferroelectric domains are energetically stable, whereas the $90^{\circ}$ ferroelectric domains are eventually stablized by $90^{\circ}$ ferroelastic domains. ${ }^{32}$ A clear switching pattern can still be observed in the VPFM image after removed the electric field for $22 \mathrm{~h}$ (Figure $2 \mathrm{q}$ ), although it was weakened somewhat due to the OP ferroelastic relaxation. However, almost no contrast was observed in the LPFM image (Figure 2r) after $22 \mathrm{~h}$; the ferroelastic domain relaxation alone IP direction was completed after $22 \mathrm{~h}$. Interestingly, a relaxation of magnetic domains also takes place as shown in Figure $2 \mathrm{~s}$, where the switched magnetic pattern nearly fades away after $22 \mathrm{~h}$. It is suggests that the E-switched magnetic domains are coupled to ferroelastic domains, which might be caused by the unique magnetic ordering status in the BTF thin film. It also reveals that the magnetic ordering is not coupling directly with ferroelectric ordering in BTF, but coupled though ferroelastic strain. The ferroelastic strain relaxation will destabilize the magneto-elastic coupling in the BTF thin film, thus, a time decay behavior of magnetic domain switching will be observed as shown in Figure 2.

During SPM imaging and quantification of local electrical properties of ferroelectric materials, there is an unavoidable issue that should be considered, that is, the effect of space charges introduced by the cantilever/tip. The resultant surface charge screening is a general feature that affects the electrostatic and transport properties of surfaces, and, thus, could probably lead to an incorrect interpretation of the data during the SPM measurements. ${ }^{33,34}$ In general, EFM is used to investigate the surface charge density $\left(\sigma_{\mathrm{s}}\right)$ of materials. The EFM image shown in Figure 21 was taken immediately after the electric poling; the bright contrast in the EFM pattern indicates that the sign of the measuring image is not determined only by the polarization 
a
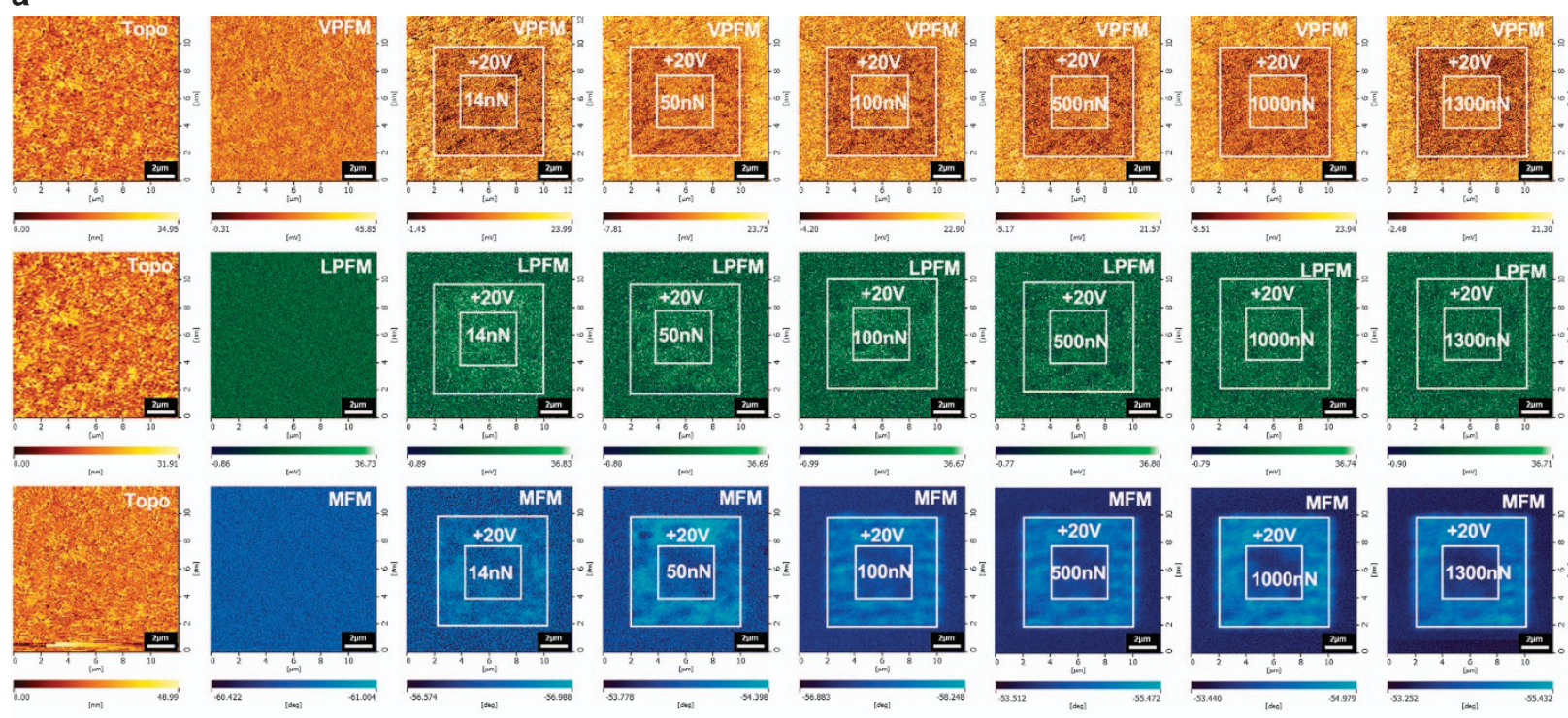

b

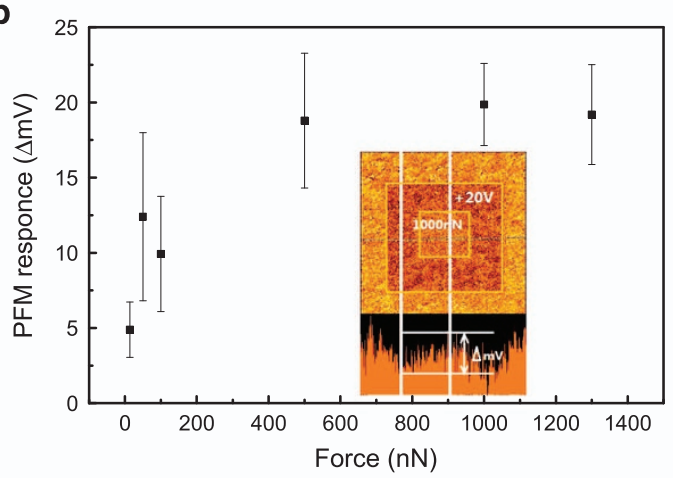

C

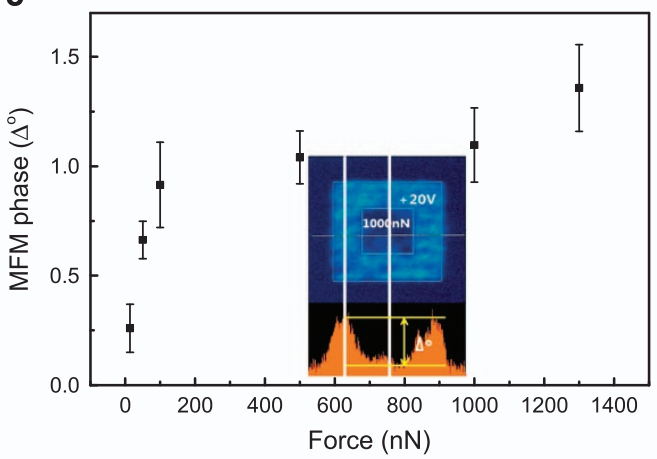

d

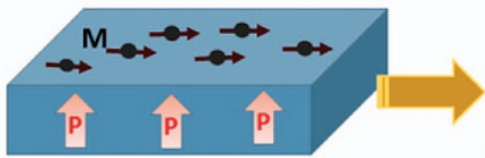

After switched by $\mathrm{E}$

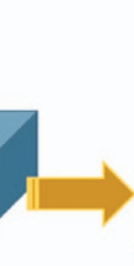

Applying mechanical force

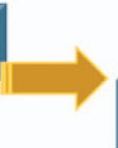

P

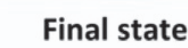

Figure 3 Mechanical force induced ferroelectric and magnetic domains switching. (a) Topographic images and corresponding vertical piezoresponse force microscope (VPFM), lateral piezoresponse force microscope (LPFM) and magnetic force microscopy (MFM) images before and after electric and mechanical switching. The $\mathrm{Bi}_{5} \mathrm{FeTi}_{3} \mathrm{O}_{15}$ (BTF) thin film that was first poled by positive electric bias of $+20 \mathrm{~V}$ over $8 \times 8 \mu \mathrm{m}^{2}$, and then switched by mechanical forces of $14,50,100,500,1000$ and $1300 \mathrm{nN}$ in the center over $4 \times 4 \mu \mathrm{m}^{2}$, respectively; (b) the surface potential $(\Delta V$ as a function of $\mathrm{F}$; (c) magnetic response $\left(\Delta^{\circ}\right)$ as a function of $F$; (d) schematic illustration of ferroelectric domain switching and magnetic domain switching when applying mechanical force.

charges but also by uncompensated charges due to charge injection by electric poling. In this work, we do not apply d.c. voltage during EFM scanning, so that the EFM image arises only from variation in the absolute value of electric field above the sample surface. ${ }^{35}$ The surface charge distribution is also recorded after remove the $E$ for $1 \mathrm{~h}$ (Figure $2 p$ ). The screening effect that because of the adsorption of charge species from the ambient significantly reduces the surface mobile charges once remove the $E$, and the charges are compensated completely after removed the $E$ for $2 \mathrm{~h}$ (Figure $2 \mathrm{t}$ ). We also carried out experiment to exclude the effect of screening charge on PFM images as shown in Supplementary Figure S6.
Domain switching by mechanical force

To investigate the influence of mechanical force on both the ferroelectric and magnetic domains of our BTF film, we have applied a set of vertical loading forces on the film. As shown in Figure 3a, the BTF thin film was firstly poled by $+20 \mathrm{~V}$ in an area of $8 \times 8 \mu \mathrm{m}^{2}$ to obtain an uniformly arranged 'mono-domain'; then the sample was scanned under a series of loading forces of 14, 50, 100, 500, 1000 and $1300 \mathrm{nN}$, respectively, in an area of $4 \times 4 \mu \mathrm{m}^{2}$ in the center; finally, the switched area was read by scanning a larger area of $12 \times 12 \mu \mathrm{m}^{2}$ at $5 \mathrm{kHz}$ in VPFM, LPFM and MFM mode. As shown in Figure 3a, for the VPFM images, when the tip force $(F)$ is $<100 \mathrm{nN}$, no obvious switching is 
observed in the 'force switching' area in the center. Further increasing $F$, the contrast between the area that is switched by $E$ and $F$ becomes more obvious. As the tip force is increased to $1300 \mathrm{nN}$, although the brightest contrast is obtained, some nonswitched domains are still exit in the center of VPFM, indicating an incomplete polarization reversal in the mechanical force switched region. The LPFM images show different switching phenomenon compared with VPFM images: a bright pattern is obtained in the E-poled region that is in accordance with Figure 2; however, the loading force seems have little effect on the ferroelectric domains along IP direction because no obvious domain switching observed in the F-poled region in the center, we consider that the sign of IP projection of the ferroelastic strain is opposite with the IP projection of piezostrain generated by external mechanical force and the magnitude is bigger than the latter, so that the back-switching of ferroelastic domain becomes more obvious in the E-switched area. For the MFM images, the magnetic domain switching become more obvious with increasing the $F$. We applied the $E$ and $F$ repeatedly in the same area, the $E$-switched and $F$-switched pattern in MFM become more homogeneous when applied force is up to $1300 \mathrm{nN}$. When the $F$ increased to $1300 \mathrm{nN}$, the magnetic domains in the F-poled region were mainly switched, indicated that the magnetization could be controlled by external mechanical force. The increasing trend towards switching of magnetic domains with increasing the load infers an enhancement of magneto-elastic coupling with increasing tip stress in BTF thin film. We did not apply higher forces because a higher force would cause wear on the tip, and the image would become blurred. The surface potential $(\Delta V)$ in PFM and magnetic response in MFM as a function of applying $F$ was recorded in Figure $3 \mathrm{~b}$ and $\mathrm{c}$, the results were acquired based on a mean square error for five times. A transition is observed in both PFM and MFM response when applying mechanical force of $100 \mathrm{nN}$, infers that the domain switching energy barrier is around $100 \mathrm{nN}$. According to the above-mentioned experimental results, both ferroelectric and magnetic domains are switched by $F$, indicating that the tip stress can induce not only local ferro-electro-elastic polarization switching but also ferroelasticmagnetic domain switching. We have to note that the polarization reversal could not be observed when applying mechanical force without an initial positive dc voltage poling. In addition, no domain switching were observed if the thin film is initially poled by negative voltage. The tip stress-induced switching pattern as shown in Figure 3a is not as bright as the E-induced switching pattern in Figure 2, due to the ferroelastic domain generation during the strain relaxation when electric field and mechanical force in the film is removed. ${ }^{36}$

A mechanical force applied on a ferroelectric material such as BTF will lead to several effects: (1) piezoelectricity is a linear electromechanical interaction describes the electricity related to the electric charge separation by stress; (2) ferroelasticity that is the phase change results in a spontaneous strain generated by the stress; and (3) flexoelectricity that describes a spontaneous polarization result from the strain gradient. In this work, the tip stress generated spontaneous strain from ferroelasticity and led to a charge separation and then generated an electric field $\left(E_{\mathrm{f}}\right)$, which was comparable with the coercive field, finally switched ferroelectric domains in the sample. For a thin film in nanoscale, the flexoelectricity could not be ignored, thus $E_{\mathrm{f}}$ attributed from both piezoelectricity and flexoelectricity. The $E_{\mathrm{f}}$ gives rise to a large field gradient divergence that persists in BTF film, eventually resulting in a polarization reversal. The preferred direction of the as-deposited BTF thin film [119] (Supplementary Figure S1) is at an angle to the spontaneous polarization direction [100]. As the mechnical force was applied on the tip along OP direction that was perpendicular to the film surface, the pizeoresponse along the OP direction in the thin film is stronger than the IP. Besides the piezoelectricity, tip stress-modified elastic strain could also account for the switching of polarization from up to down. Due to the anisotropic elastic strain in BTF thin film, the strain relaxation along IP direction is faster than the OP. As shown in Supplementary Figure S4, the ferroelectric domain and magnetic domain were subsequently switched by applying $+20 \mathrm{~V}$ after the mechanical switching, indicating that the mechanical force could act similarly as an electric field on domain switched ferroelectric domain and magnetic domain.

The mechanical force introduces a piezostrain in nano-region, which modifies the original elastic strain state generated from the thermal process during film deposition, ${ }^{37}$ and results in a consequent reorientation of the local magnetism driven by the strain coupling between small magnetic volume and ferroelastic domians. ${ }^{38}$ A second possibility is that before the mechanical force is applied, the film is already under a small IP stress because of the electric poling, producing a very small strain due to the high modulus. The addition of an electrostatic potential on the top of the film can be expected to cause cation displacement, and the existence of an IP stress will ease this cation displacement, leading to a larger deformation that would have an influence on the magnetic volume in the film as well. ${ }^{35,39}$ The switching process is illustrated in Figure $3 \mathrm{~d}$. We suppose that the rotational moment and polar distortions in BTF would be coupled under certain conditions involved in strain state, as in $\mathrm{BiFeO}_{3} .{ }^{25}$ The tip stress-induced $E_{\mathrm{f}}$ changes the free energy profile asymmetrically, which would also take account of the factors for switching the domains. Further investigation on the coupling configuration between ferroelectric domains and magnetic domains would be complicated, it is necessary to discover the ME coupling mechanism in high-quality BTF single crystals.

\section{Domain switching by magnetic field}

In order to investigate the effects of magnetic field on the domain switching in BTF thin film, an alternating magnetic field $(H)$ was applied on the BTF thin film, which was carried out on an E-sweep SPM system in vacuum. Figure $4 \mathrm{a}-\mathrm{d}$ shows topography and MFM images of an as-deposited BTF thin film under the magnetic field of 0 , 50 and $100 \mathrm{mT}$, respectively. Due to the weak magnetization of the BTF thin film, no magnetic domains could be observed without applying $H$. The magnetic domains became more obvious with increasing $H$, indicating that the magnetic domains could be manipulated by $H$. Due to the limitations of dc bias in this system, the sample was poled by dc bias of $\pm 10 \mathrm{~V}$ in VPFM mode, as shown in Figure $4 \mathrm{f}$. Although the ferroelectric domains were not fully switched, the box-in-box PFM pattern was observed. The corresponding MFM image was scanned in the same area in vacuum $\left(7.1 \times 10^{-4} \mathrm{~Pa}\right)$, recognized by comparing the typical partials in Figure $4 \mathrm{i}$ and $\mathrm{j}$. When we switch from PFM to MFM mode after electric poling, we found that the image in MFM mode was 'expand' due to the recording offset after evacuation, so we enlarge the scanning area $\left(12 \times 12 \mu \mathrm{m}^{2}\right)$ to make sure fully record the E-switched image in MFM mode. We trace the same area by comparing typical structures (two particles) in topographic images as shown in Figure 4e and f. A clear switching pattern was observed, even the magnetic domains were not completely switched, and indicating the ME coupling in BTF is stable in vacuum at RT. An IP $H$ of $100 \mathrm{mT}$ was applied on the sample (schematic diagram shown in Figure $4 \mathrm{~m}$ ) to investigate the effect of $H$ on the $E$-switched domains. PFM and MFM images were collected with shift the direction of $H$ from $+100 \mathrm{mT}$ to $-100 \mathrm{mT}$ as shown in Figure $4 \mathrm{~g}$, $\mathrm{h}, \mathrm{k}$ and $\mathrm{l}$, respectively. The ferroelectric domains were rarely affected 

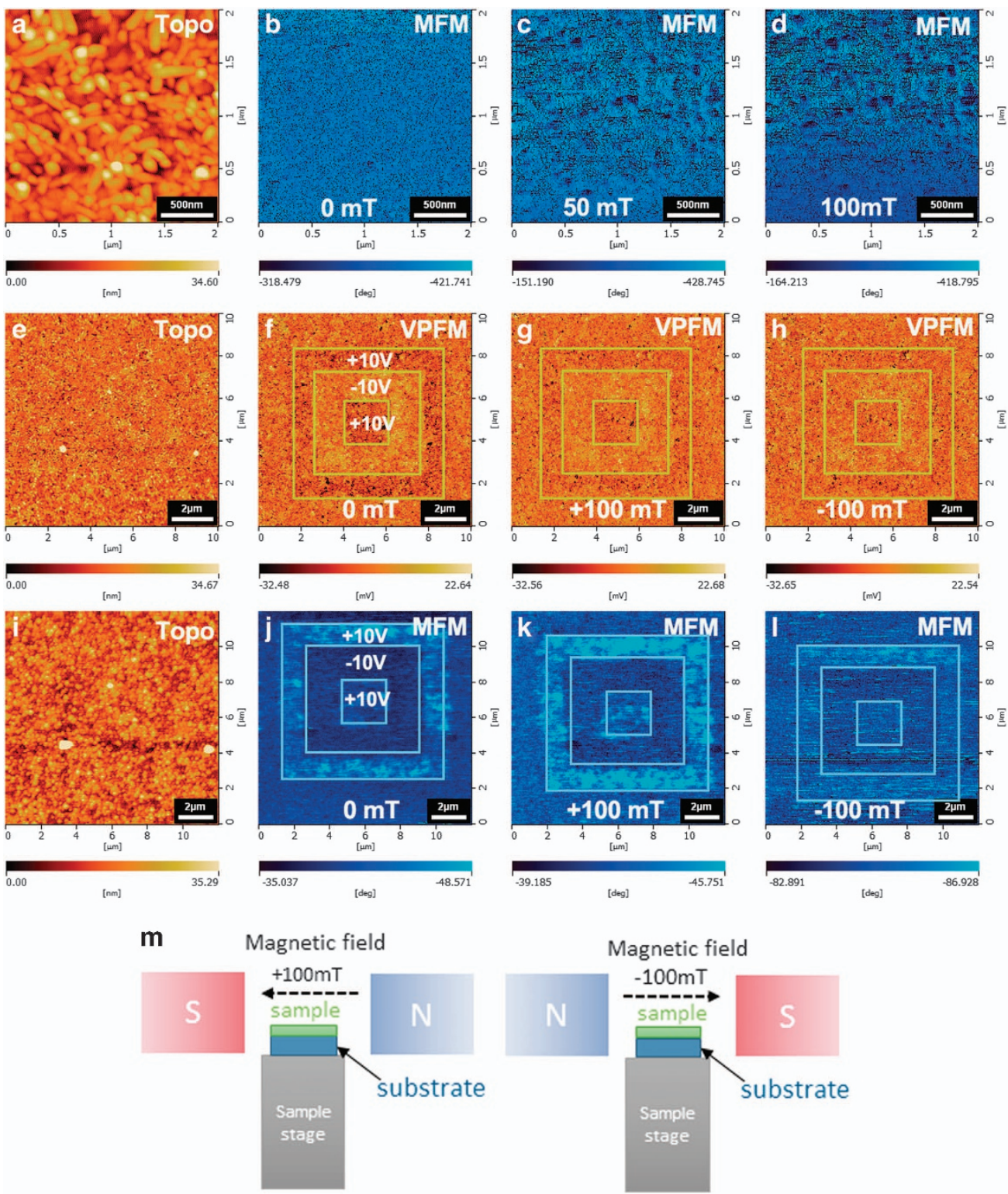

Figure 4 Control of both ferroelectric and magnetic domains by magnetic field $(H)$. Topography (a) and magnetic force microscopy (MFM) images measured under magnetic field of (b) 0 , (c) 50 and (d) $100 \mathrm{mT}$, with the part in the white ellipse showing a typical phenomenon: the magnetic domains become more apparent with increasing magnetic field; (e) topography and (f) vertical piezoresponse force microscope (VPFM) image of the $\mathrm{Bi}_{5} \mathrm{FeTi}_{3} \mathrm{O}_{15}$ (BTF) thin film that was first poled by electric field; corresponding (i) topography and (j) MFM image measured in vacuum. (g) VPFM image and (k) MFM image measured under magnetic field of $+100 \mathrm{mT}$. (h) VPFM image and (I) MFM image under $-100 \mathrm{mT}$. (m) Schematic diagram of in-plane magnetic field applied on the BTF thin film. The signs ' + ' and ' - ' for the magnetic field represent different magnetic field direction.

by $+100 \mathrm{mT}$ (Figure $4 \mathrm{~g}$ ), whereas the magnetic domains were enhanced by an $H$ of $+100 \mathrm{mT}$ (Figure 4k), which indicated that the $+100 \mathrm{mT}$ field lied in accordance with the easy axis of BTF helps complete the switching of magnetic domains that were incompletely switched by $E$. When the IP $H$ was applied in the reverse direction (marked as $-100 \mathrm{mT}$ ), the magnetic domains were switched off (Figure 41). However, some of the switched ferroelectric domains were switched back when the direction of the direction of $H$ was changed to $-100 \mathrm{mT}$ (Figure 4h), and this result indicates that the $H$ in this direction helps reducing the back-switching energy of ferroelectric domains. As we have discussed above that the back-switching of ferroelectric domains is mainly due to the strain relaxation of ferroelastic domains, this phenomenon infers a unique magneto-elasto coupling behavior in the BTF thin film, indicating that the coupling is highly depend on the symmetric orientation. The magnetization switching can also switch the ferroelectric polarization through ferroelastic strain. Reverse process could be also expected as follows: the magnetic $\mathrm{Fe}^{3+}$ ordering is relative to the polarization in the BTF film. The $H$ forces a rotation of $\mathrm{Fe}^{3+}$ spins that leads to a symmetry distortion of the crystal structure, and changes the strain state in the film, thereby controls the polarization of the BTF film.

\section{CONCLUSION}

In summary, we have demonstrated the manipulation of both ferroelectric and magnetic domains by electric field, magnetic field 
and mechanical force in a BTF thin film at RT. However, due to the relaxation of ferroelastic domains in the BTF film and possible short-range magnetic ordering in Fe-rich nano-regions, the magneto-elastic coupling is not stable, $E$-induced magnetic domain switching is fade away after the electric field is removed. The successful manipulation of both ferroelectric domain and magnetic domain by mechanical force proved the switching path role of ferroelastic strain in the ME coupling in the BTF thin film, which would be important for further understanding the magneto-elastic-electric coupling in multiferroics. The mechanical switching of both ferroelectric and magnetic domain behavior in multiferroics shows a complex intercoupling among polarization, magnetization and strain, and provides the possibility of manipulating one physical parameter by using one of the three external stimuli: magnetic field, electrical field and mechanical force, which could yield an additional degree of freedom in novel multifunctional device design. The mechanical switching of both ferroelectric domains and magnetic domains gives rise to an enormous potential application in high-density data storage via mechanical means, and might help to overcome the leakage or dielectric breakdown as in direct electric switching.

\section{CONFLICT OF INTEREST}

The authors declare no conflict of interest.

\section{ACKNOWLEDGEMENTS}

Part of this work was supported by a Green Network of Excellence (GRENE) project of the Ministry of Education, Culture, Sports, Science and Technology (MEXT) of Japan. Author TT Jia is grateful for support by grants from the Japan Society for the Promotion of Science (JSPS; no. 26-04206). Author ZX Cheng thanks the Australian Research Council (ARC) for support through a Future Fellowship. Author H.Y. Zhao thanks the National Natural Science Foundation of China for support (No. 51402327). We acknowledge Mrs M. Nakabayashi at GRENE for transmission electron microscope observations. A part of this work was conducted in the Research Hub for Advanced Nano Characterization, The University of Tokyo, supported by MEXT, Japan. Part of this work was also supported by the 'Nanotechnology Platform' (project no. 12024046) sponsored by MEXT, Japan. We gratefully acknowledge the help of Dr Ehsan Nasr Esfahani and Professor Jiangyu Li in University of Washington for discussion.

1 Eerenstein, W., Mathur, N. D. \& Scott, J. F. Multiferroic and magnetoelectric materials. Nature 442, 759-765 (2006).

2 Scott, J. F. Room-temperature multiferroic magnetoelectrics. NPG Asia Mater. 5, e72 (2013).

3 Cheong, S. W. \& Mostovoy, M. Multiferroics: a magnetic twist for ferroelectricity. Nat. Mater. 6, 13-20 (2007).

4 Spaldin, N. A. \& Fiebig, M. The renaissance of magnetoelectric multiferroics. Science 309, 391-392 (2005).

5 Hehn, M., Ounadjela, K., Bucher, J.-P., Rousseaux, F., Decanini, D., Bartenlian, B. \& Chappert, C. Nanoscale magnetic domains in mesoscopic magnets. Science 272, 1782-1785 (1996)

6 Geng, Y. N., Lee, N., Choi, Y. J., Cheong, S. W. \& Wu, W. D. Collective magnetism at multiferroic vortex domain walls. Nano Lett. 12, 6055-6059 (2012).

7 Zhao, T., Scholl, A., Zavaliche, F., Lee, K., Barry, M., Doran, A., Cruz, M. P., Chu, Y. H., Ederer, C., Spaldin, N. A., Das, R. R., Kim, D. M., Baek, S. H., Eom, C. B. \& Ramesh, R. Electrical control of antiferromagnetic domains in multiferroic $\mathrm{BiFeO}_{3}$ films at room temperature. Nat. Mater. 5, 823-829 (2006).

8 Keeney, L., Maity, T., Schmidt, M., Amann, A., Deepak, N., Petkov, N., Roy, S., Pemble, M. E., Whatmore, R. W. \& Johnson, D. Magnetic field-induced ferroelectric switching in multiferroic aurivillius phase thin films at room temperature. J. Am. Ceram. Soc. 96, 2339-2357 (2013).

9 Lu, H., Bark, C. W., Esque de los Ojos, D., Alcala, J., Eom, C. B., Catalan, G. \& Gruverman, A. Mechanical writing of ferroelectric polarization. Science 336, 59-61 (2012).
10 Zhe, Z., Xue Jun, Z., Dan Shu, Z. \& Ju, G. Threshold stress of $90^{\circ}$ domain switching in the misfit strain-external stress phase diagram. Jpn J. Appl. Phys. 53, 125501 (2014).

11 Sharma, P., Ryu, S., Viskadourakis, Z., Paudel, T. R., Lee, H., Panagopoulos, C., Tsymbal, E. Y., Eom, C.-B. \& Gruverman, A. Electromechanics of ferroelectric-like behavior of $\mathrm{LaAlO}_{3}$ thin films. Adv. Funct. Mater. 25, 6538-6544 (2015)

12 Kim, D. J., Paudel, T. R., Lu, H., Burton, J. D., Connell, J. G., Tsymbal, E. Y., Ambrose Seo, S. S. \& Gruverman, A. Room-temperature ferroelectricity in hexagonal $\mathrm{TbMnO}_{3}$ thin films. Adv. Mater. 26, 7660-7665 (2014).

$13 \mathrm{Heo}$, Y., Jang, B.-K., Kim, S. J., Yang, C.-H. \& Seidel, J. Nanoscale mechanical softening of morphotropic $\mathrm{BiFeO}_{3}$. Adv. Mater. 26, 7568-7572 (2014).

14 Zhao, H. Y., Dou, S. X., Liu, Y., Kimura, H., Yu, J. D., Cheng, Z. X., Matsumoto, T., Osada, M., Tohei, T., Wang, J. L., Shibata, N., Wang, X. L. \& Ikuhara, Y. Large magnetoelectric coupling in magnetically short-range ordered $\mathrm{Bi}_{5} \mathrm{Ti}_{3} \mathrm{FeO}_{15}$ film. Sci. Rep. 4, 5255 (2014).

15 Newnham, R. E., Wolfe, R. W. \& Dorrian, J. F. Structural basis of ferroelectricity in the bismuth titanate family. Mater. Res. Bull. 6, 1029-1039 (1971).

16 Snedden, A., Hervoches, C. \& Lightfoot, P. Ferroelectric phase transitions in $\mathrm{SrBi}_{2} \mathrm{Nb}_{2} \mathrm{O}_{9}$ and $\mathrm{Bi}_{5} \mathrm{Ti}_{3} \mathrm{FeO}_{15}$ : a powder neutron diffraction study. Phys. Rev. B 67, 092102 (2003).

17 Bai, W., Zhu, J. Y., Wang, J. L., Lin, T., Yang, J., Meng, X. J., Tang, X. D., Zhu, Z. Q. \& Chu, J. H. Effects of annealing temperature on the structures, ferroelectric and magnetic properties of Aurivillius $\mathrm{Bi}_{5} \mathrm{Ti}_{3} \mathrm{FeO}_{15}$ polycrystalline films. J. Magn. Magn. Mater. 324, 2265-2270 (2012).

18 Watanabe, T. \& Funakubo, H. Controlled crystal growth of layered-perovskite thin films as an approach to study their basic properties. J. Appl. Phys. 100, 051602 (2006).

19 Chen, A. P., Zhou, H. H., Bi, Z. X., Zhu, Y. Y., Luo, Z. P., Bayraktaroglu, A., Phillips, J., Choi, E. M., MacManus-Driscoll, J. L., Pennycook, S. J., Narayan, J., Jia, Q. X., Zhang, X. H. \& Wang, H. Y. A new class of room-temperature multiferroic thin films with bismuth-based supercell structure. Adv. Mater. 25, 1028-1032 (2013).

20 Noguchi, Y., Miyayama, M. \& Kudo, T. Direct evidence of A-site-deficient strontium bismuth tantalate and its enhanced ferroelectric properties. Phys. Rev. B 63, 214102 (2001).

21 Yang, F. J., Su, P., Wei, C., Chen, X. Q., Yang, C. P. \& Cao, W. Q. Large magnetic response in $\left(\mathrm{Bi}_{4} \mathrm{Nd}\right) \mathrm{Ti}_{3}\left(\mathrm{Fe}_{0.5} \mathrm{Co}_{0.5}\right) \mathrm{O}_{15}$ ceramic at room-temperature. J. Appl. Phys. 110, 126102 (2011).

22 Mao, X., Wang, W., Chen, X. \& Lu, Y. Multiferroic properties of layer-structured $\mathrm{Bi}_{5} \mathrm{Fe}_{0.5} \mathrm{Co}_{0.5} \mathrm{Ti}_{3} \mathrm{O}_{15}$ ceramics. Appl. Phys. Lett. 95, 082901 (2009).

23 Singh, R. S., Bhimasankaram, T., Kumar, G. S. \& Suryanarayana, S. V. Dielectric and magnetoelectric properties of $\mathrm{Bi}_{5} \mathrm{FeTi}_{3} \mathrm{O}_{15}$. Solid State Commun. 91, 567-569 (1994).

24 Jaafar, M., Iglesias-Freire, O., Serrano-Ramon, L., Ibarra, M. R., de Teresa, J. M. \& Asenjo, A. Distinguishing magnetic and electrostatic interactions by a Kelvin probe force microscopy-magnetic force microscopy combination. Beilstein J. Nanotechnol. 2, 552-560 (2011).

25 Ederer, C. \& Spaldin, N. Weak ferromagnetism and magnetoelectric coupling in bismuth ferrite. Phys. Rev. B 71, 060401 (R) (2005).

26 Kalinin, S. V., Rodriguez, B. J., Borisevich, A. Y., Baddorf, A. P., Balke, N., Chang, H. J., Chen, L. Q., Choudhury, S., Jesse, S., Maksymovych, P., Nikiforov, M. P. \& Pennycook, S. J. Defect-mediated polarization switching in ferroelectrics and related materials: from mesoscopic mechanisms to atomistic control. Adv. Mater. 22, 314-322 (2010).

27 Rondinelli, J. M., Stengel, M. \& Spaldin, N. A. Carrier-mediated magnetoelectricity in complex oxide heterostructures. Nat. Nanotechnol. 3, 46-50 (2008).

28 Baek, S. H., Jang, H. W., Folkman, C. M., Li, Y. L., Winchester, B., Zhang, J. X., He, Q., Chu, Y. H., Nelson, C. T., Rzchowski, M. S., Pan, X. Q., Ramesh, R., Chen, L. Q. \& Eom, C. B. Ferroelastic switching for nanoscale non-volatile magnetoelectric devices. Nat. Mater. 9, 309-314 (2010).

29 Ahn, S.-I., Noguchi, Y., Miyayama, M. \& Kudo, T. Structural and electrical characterization of $\mathrm{Bi}_{5} \mathrm{Ti}_{3} \mathrm{Fe}_{1-x} \mathrm{Mn}_{\mathrm{x}} \mathrm{O}_{15}$ system. Mater. Res. Bull. 35, 825-834 (2000).

30 Jartych, E., Mazurek, M., Lisińska-Czekaj, A. \& Czekaj, D. Hyperfine interactions in some Aurivillius $\mathrm{Bi}_{\mathrm{m}+1} \mathrm{Ti}_{3} \mathrm{Fe}_{\mathrm{m}-3} \mathrm{O}_{3 \mathrm{~m}+3}$ compounds. J. Magn. Magn. Mater. 322, 51-55 (2010).

31 Jartych, E., Pikula, T., Mazurek, M., Lisinska-Czekaj, A., Czekaj, D., Gaska, K., Przewoznik, J., Kapusta, C. \& Surowiec, Z. Antiferromagnetic spin glass-like behavior in sintered multiferroic Aurivillius $\mathrm{Bi}_{m+1} \mathrm{Ti}_{3} \mathrm{Fe}_{m-3} \mathrm{O}_{3 m+3}$ compounds. J. Magn. Magn. Mater. 342, 27-34 (2013).

32 Chen, L., Ouyang, J., Ganpule, C. S., Nagarajan, V., Ramesh, R. \& Roytburd, A. L. Formation of $90^{\circ}$ elastic domains during local $180^{\circ}$ switching in epitaxial ferroelectric thin films. Appl. Phys. Lett. 84, 254-256 (2004).

33 Cuniot-Ponsard, M. Kelvin probe force microscopy and electrostatic force microscopy responses to the polarization in a ferroelectric thin film: theoretical and experimental investigations. J. Appl. Phys. 114, 014302 (2013).

34 Morozovska, A. N., Eliseev, E. A., Svechnikov, G. S. \& Kalinin, S. V. Nanoscale electromechanics of paraelectric materials with mobile charges: Size effects and nonlinearity of electromechanical response of $\mathrm{SrTiO}_{3}$ films. Phys. Rev. B 84, 045402 (2011).

35 Yan, F., Chen, G. N., Lu, L., Finkel, P. \& Spanier, J. E. Local probing of magnetoelectric coupling and magnetoelastic control of switching in $\mathrm{BiFeO}_{3}-\mathrm{CoFe}_{2} \mathrm{O}_{4}$ thin-film nanocomposite. Appl. Phys. Lett. 103, 042906 (2013). 
36 Wang, C., Ke, X., Wang, J., Liang, R., Luo, Z., Tian, Y., Yi, D., Zhang, Q., Wang, J., Han, X.-F., Van Tendeloo, G., Chen, L.-Q., Nan, C.-W., Ramesh, R. \& Zhang, J. Ferroelastic switching in a layered-perovskite thin film. Nat. Commun. 7, 10636 (2016).

37 Yudin, P. V. \& Tagantsev, A. K. Fundamentals of flexoelectricity in solids. Nanotechnology 24, 432001 (2013).

38 Shirahata, Y., Shiina, R., González, D. L., Franke, K. J. A., Wada, E., Itoh, M., Pertsev, N. A., van Dijken, S. \& Taniyama, T. Electric-field switching of perpendicularly magnetized multilayers. NPG Asia Mater. 7, e198 (2015).

39 Birenbaum, A. Y. \& Ederer, C. Potentially multiferroic Aurivillius phase $\mathrm{Bi}_{5} \mathrm{FeTi}_{3} \mathrm{O}_{15}$ : cation site preference, electric polarization, and magnetic coupling from first principles. Phys. Rev. B 90, 214109 (2014). (c) (i) This work is licensed under a Creative Commons Attribution 4.0 International License. The images or other third party material in this article are included in the article's Creative Commons license, unless indicated otherwise in the credit line; if the material is not included under the Creative Commons license, users will need to obtain permission from the license holder to reproduce the material. To view a copy of this license, visit http:// creativecommons.org/licenses/by/4.0/

(C) The Author(s) 2017

Supplementary Information accompanies the paper on the NPG Asia Materials website (http://www.nature.com/am) 